\title{
Cardiac tamponade: appearance on CT
}

\author{
Cicero T. Silva $\cdot$ Kenneth Baker
}

Received: 19 January 2009 / Accepted: 11 February 2009 / Published online: 10 March 2009

(C) Springer-Verlag 2009

A 16-year-old boy with a history of cardiac catheter ablation collapsed while wrestling. Following admission to the emergency department he underwent a contrastenhanced CT scan of the chest (Fig. 1) and abdomen (Fig. 2). This showed a pericardial effusion, bowing of the interventricular septum, periportal edema, ascites, and IVC and right renal vein enlargement. Shortly after, he became hemodynamically unstable and underwent a pericardiocentesis with drainage of blood. He died a few hours after admission to the ICU.

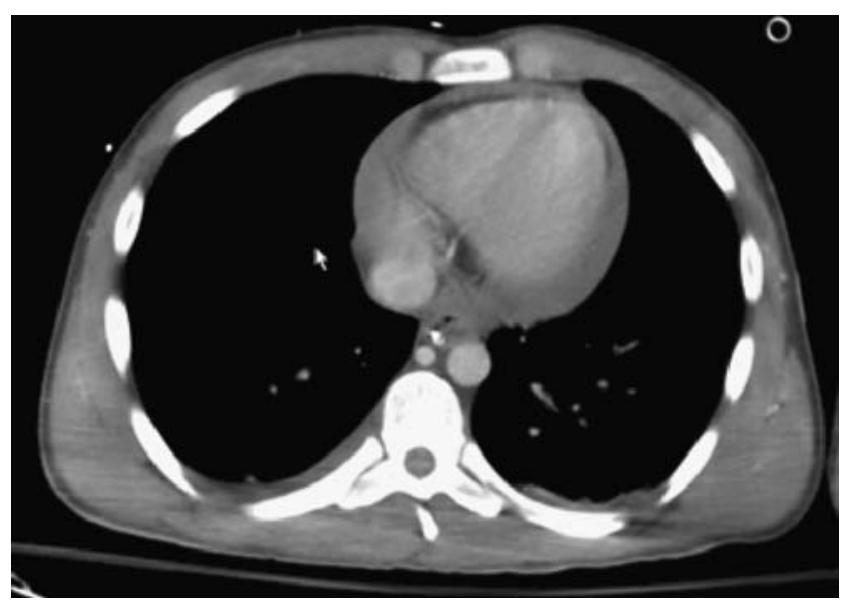

Fig. 1 Chest CT image at the level of the ventricles shows pericardial effusion and interventricular septum bowing

\footnotetext{
C. T. Silva $(\bowtie) \cdot K$. Baker

Department of Diagnostic Radiology,

Yale University School of Medicine,

20 York Street South, Pavilion 2nd Floor,

New Haven, CT 06510-3220, USA

e-mail: cicero.torres@gmail.com
}

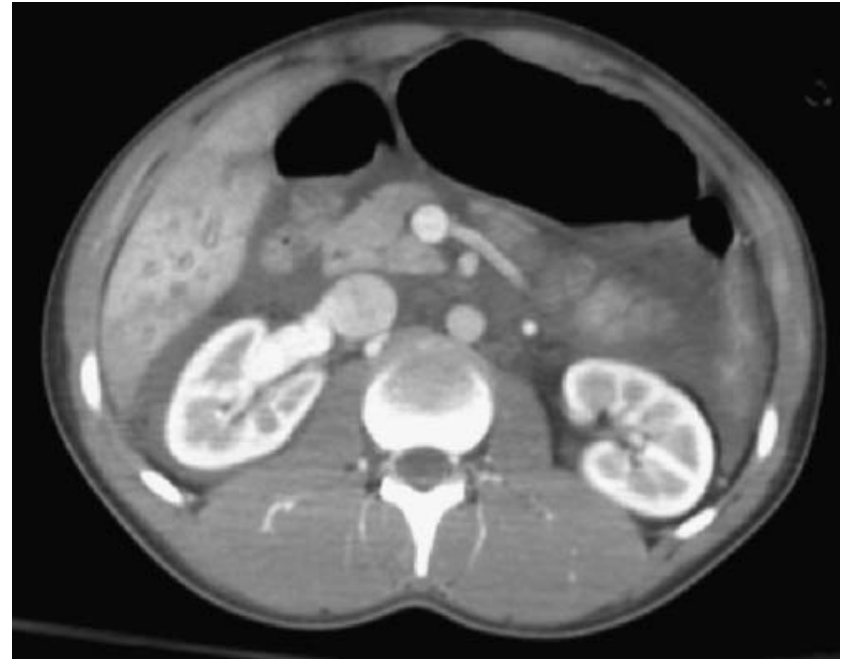

Fig. 2 Abdominal CT image at the renal level shows periportal edema, ascites, IVC and right renal vein enlargement

Cardiac tamponade results from heart compression and diminished cardiac output secondary to accumulation of fluid, pus, blood, gas or tissue within the pericardial cavity [1]. CT findings include pericardial effusion, cardiac chamber deformity, interventricular septum bowing, SVC and IVC distension, contrast medium reflux into the azygos vein and IVC, hepatic and renal vein enlargement, periportal edema and ascites [1,2].

\section{References}

1. Restrepo CS, Lemos DF, Lemos JA et al (2007) Imaging findings in cardiac tamponade with emphasis on CT. Radiographics 27:1595-1610

2. Huang C-H, Liu C-L, Chen W-K (2009) Periportal edema and ascites: computed tomographic signs of traumatic cardiac tamponade. Am J Emerg Med 27:127.e3-127.e4 